\title{
ANÁLISE DA VARIAÇÃO DAS PROPRIEDADES FÍSICAS DOS GRÃOS DE SOJA DURANTE O PROCESSO DE SECAGEM
}

\author{
Deise M. RIBEIRO , Paulo C. CORREA', Diana H. RODRIGUES ${ }^{2}$, André L. D. GONELI
}

\begin{abstract}
RESUMO
O presente trabalho foi realizado com o objetivo de se avaliar o efeito da secagem nas propriedades fisicas dos grãos de soja, e analisar as diferentes metodologias de determinação da massa específica aparente e da massa específica real. Alèm disso, avaliaramse a contração volumétrica dos grãos de soja durante a secagem e, ajustes de diferentes modelos matemáticos aos valores experimentais das propriedades analisadas, em função do teor de água. Com base nos resultados obtidos, concluiu-se que a redução do teor de água provoca diminuição linear da porosidade na faixa entre 0,31 e 0,15 (b.s.) e aumento das massas específicas aparente e real. Além disso, os resultados obtidos para massa específica real e aparente, pelo método de determinação com base nos constituintes dos grãos de soja, apresentaram-se inferiores aos valores experimentais. Os modelos de BALA \& WOODS [2] adaptado, RAHMAN, linear e exponencial são apropriados para descrição da contração volumétrica da massa de grãos de soja, enquanto o modelo de BALA \& WOODS [2] adaptado é o mais apropriado para descrição da contração volumétrica unitária da soja.

Palavras-chave: porosidade; massa específica aparente e real; contração volumétrica; modelagem.
\end{abstract}

\section{SUMMARY}

ANALYSIS OF PHYSICAL PROPERTIES VARIATION OF SOY GRAINS DURING THE DRYING PROCESS. The present work was accomplished with the objective of evaluating the drying effect on soy grain physical properties and to analyze different methodologies for determining bulk density and specific gravity. Also, the soy grain shrinkage was evaluated during the drying and different mathematical models were fitted to the experimental data in order to analyze the properties in connection with their moisture content. Based on the obtained results it can be concluded that the reduction in the moisture content promotes the linear decrease in the porosity in the range from 0,31 to 0,15 (b.s.) and increase of both the bulk density and specific gravity. Also the soy grain bulk density and specific gravity values found through the grain chemical composition were lower than the experimental data. The adapted BALA \& WOODS [2], RAHMAN, linear and exponential models are appropriate for description of the soy bulk grain shrinkage, while only the adapted BALA \& WOODS [2] model is the most appropriate for description of the soy unitary grain shrinkage.

Keywords: porosity; bulk density; specific gravity; shrinkage; mathematical modeling.

\section{1 - INTRODUÇÃO}

A soja é um produto com grande expressão na economia externa e interna do Brasil, não só pelo seu valor como grão para consumo, mas pelas grandes possibilidades de utilização devido aos seus altos teores de óleo e proteína e, também, à boa valorização comercial de seus resíduos.

A produção de grãos com elevada qualidade requer, entre outras recomendações, que o produto seja colhido sadio e antecipadamente, visando minimizar as perdas ocasionadas no campo pelos ataques de insetos e microrganismos. Dessa maneira, devido ao teor de água elevado por ocasião da colheita, a secagem constitui uma das operações de primordial importância entre as técnicas envolvidas na conservação das qualidades desejáveis de produtos de origem vegetal.

Recebido para publicação em 24/05/2005. Aceito para publicação em 11/08/2005(001535).

Universidade Federal de Vicosa. Caixa Postal: 270. CEP: 36571 o00, Viçosa-MG, Brasil.E-mail:deise_eng@yahoo.com.br A quem a correspondência deve ser enviada.
A redução do teor de água dos grãos envolve simultaneamente processos de transferência de calor e massa, o que pode alterar de forma substancial a qualidade e as propriedades fisicas do produto, dependendo do método e das condições de secagem [9].

O conhecimento das propriedades físicas dos produtos agrícolas não tem uso restrito à engenharia, podendo as informações ser de grande utilidade em outros ramos da ciência ou tecnologia relacionadas com o comportamento fisico e processamento de frutos e vegetais, de um modo geral. As principais aplicações desses conhecimentos se destinam ao dimensionamento adequado de máquinas utilizadas no processamento e melhor caracterização do produto [15].

As propriedades fisicas dos grãos são características relevantes na otimização dos processos industriais e no desenvolvimento de novos projetos e equipamentos utilizados nas operações pós-colheita. Variações destas propriedades, em função do teor de água e de outros fatores durante a secagem de vários produtos, têm sido investigadas por diversos autores [7, 14, 19].

A massa específica real ou unitária de partículas sólidas granulares, tais como grãos e cereais, pode ser estimada, de acordo com LEWIS [13], a partir do conhecimento da composição química do produto.

Durante o processo de secagem podem ocorrer alterações nas propriedades físicas e químicas da soja, afetando assim o seu potencial como matéria-prima para fa- 
bricação de determinados produtos. A representação matemática do processo de secagem de diversos produtos agrícolas há algum tempo vem sendo estudada e utilizada na tentativa de predizer os fenômenos que acontecem durante este processo.

A maioria dos modelos empregados para representar a secagem dos produtos agrícolas foi desenvolvida negligenciando-se a contração volumétrica do produto durante o processo de desidratação [4]. Estas equações vêm sendo revisadas para incorporar o efeito desse fenômeno para uma melhor simulação do processo e precisão dos resultados [11].

Mudanças volumétricas dos produtos, devido a sua desidratação, são relatadas como sendo as principais causas das alterações das principais propriedades fisicas de produtos agrícolas $[17,21]$.

RATTI [17] e ZOGZAS, MAROULIS \& KOURIS [23] observaram que a contração volumétrica de produtos vegetais durante a secagem não é função exclusiva do teor de umidade, mas também, dependente das condições do processo e da geometria do produto.

As bases teóricas para o conhecimento do processo de contração volumétrica envolvem complexas leis mecânicas e de deformação de materiais [21]. No entanto, muitos pesquisadores têm utilizado aproximações e modelos empíricos na tentativa de melhor representar esse complicado fenômeno em produtos de natureza biológica [10, 12].

Considerando o exposto e a grande importância e variabilidade existente nas propriedades fisicas dos produtos agrícolas, o presente trabalho teve como objetivo avaliar o efeito da secagem nas propriedades físicas dos grãos de soja, bem como analisar as diferentes metodologias de determinação da massa específica aparente e da massa específica real. Objetivou-se também avaliar a contração volumétrica dos grãos de soja durante a secagem, além de ajustar diferentes modelos matemáticos aos valores experimentais das propriedades analisadas, em função do teor de água.

\section{2 - MATERIAL E MÉTODOS}

O presente trabalho foi realizado no Laboratório de Propriedades Físicas e Avaliação de Qualidade de Produtos Agrícolas do Centro Nacional de Treinamento em Armazenagem (CENTREINAR), localizado no Campus da Universidade Federal de Viçosa (UFV), Viçosa - MG.

Utilizaram-se grãos de soja, variedade UFV 20, colhidos com teor de água de aproximadamente $45 \%$ base seca, posteriormente secos em câmaras do tipo B.O.D., com temperatura de $35^{\circ} \mathrm{C}$, e teor de água variando de 45 a $12 \%$ b.s.

Os teores de água do produto foram determinados pelo método da estufa, $105 \pm 1^{\circ} \mathrm{C}$, durante $24 \mathrm{~h}$, em três repetições [3].

Ao término de cada tratamento de secagem as amos- tras foram homogeneizadas e encaminhadas para determinação das propriedades físicas.

\section{1 - Porosidade}

A porosidade da massa granular foi determinada, pela média de três repetições, utilizando um picnômetro de comparação a ar (Figura 1) construído no Departamento de Engenharia Agrícola da Universidade Federal de Viçosa, segundo o processo descrito por DAY, citado por MOHSENIN [15].

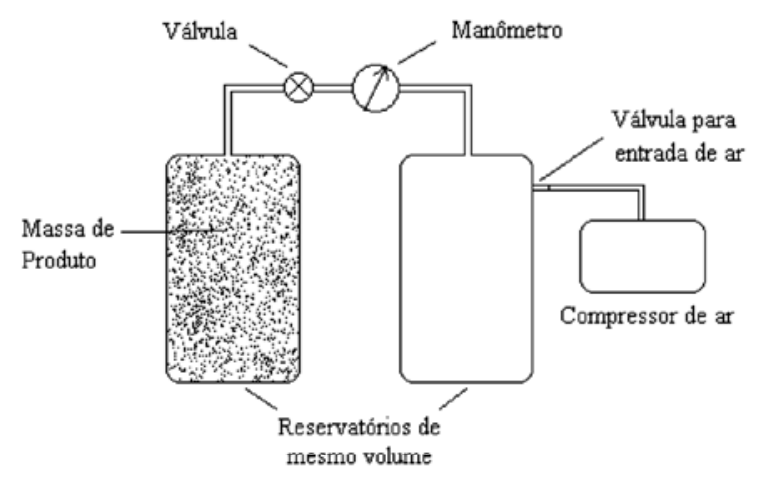

FIGURA 1 - Desenho esquemático do picnômetro

\section{2 - Massa especifica aparente e real}

A massa específica aparente ( $\rho$ ap1) foi determinada utilizando-se uma balança de peso hectolitro da marca Dallemole, com capacidade de um litro, em três repetições para cada tratamento. A massa específica real $\left(\rho_{t}\right)$ foi obtida em função da porosidade ( $(\varepsilon)$ e da massa específica aparente $\left(\rho_{\ldots}\right)$ de acordo com a equação descrita por

$$
\begin{aligned}
& P_{\mathrm{rl}}=\frac{P_{\mathrm{apl}}}{(1-\varepsilon)} \\
& \text { MOHSENIN [15]: }
\end{aligned}
$$

Os resultados experimentais das massas específicas aparente e real foram comparados aos obtidos pelo cálculo a partir da composição química média dos grãos de trigo ( $\rho_{\mathrm{r} 2}$ e $\rho_{\mathrm{c}, 2}$, respectivamente), adaptando a metodologia descrita por LEWIS [13], de acordo com a seguinte equação:

$p=\frac{1+U^{*}}{\left(U^{*} / \rho_{a g}\right)+\left(\omega_{1} / \rho_{1}\right)+\cdots+\left(\omega_{n} / \rho_{n}\right)}$

em que:

$$
\begin{aligned}
& \rho_{\text {s. }} \quad \text { - massa específica da água, } 1000 \mathrm{~kg} \mathrm{~m} \\
& \text { U - teor de água do produto, decimal (base seca) } \\
& \rho_{1} \ldots \rho_{\text {n }} \quad \text { - massa específica dos constituintes de } 1 \mathrm{an}, \mathrm{kg} \mathrm{m} \\
& \omega_{1} \ldots \omega_{n} \text { - teores dos constituintes do produto de } 1 \text { a } \mathrm{n} \text {, } \\
& \text { decimal (base seca) }
\end{aligned}
$$


Foram utilizados os teores médios dos constituintes dos grãos de trigo segundo a USDA [22] (Tabela 1).

TABELA 1 - Composição química média de $100 \mathrm{~g}$ de grãos de soja

\begin{tabular}{lc}
\hline \multicolumn{1}{c}{ Constituinte } & Participação na Composição Química $(\mathrm{mg})$ \\
\hline Água & $8.540,0$ \\
Proteina & $36.490,0$ \\
Carboidratos (Totais) & $30.160,0$ \\
Vitaminas & 10,537 \\
Lipidios (Totais) & $19.940,0$ \\
Minerais & \\
Cálcio & 277,0 \\
Fósforo & 704,0 \\
Ferro & 15,7 \\
Potássio & $1.797,0$ \\
Cobre & 1,658 \\
Manganês & 2,517 \\
Magnésio & 280,0 \\
Sódio & 2,0 \\
Zinco & 4,89 \\
\hline
\end{tabular}

\section{3 - Contração volumétrica unitária e da massa de grãos}

A contração volumétrica unitária $\left(\psi_{\star}\right)$ e da massa de grãos $\left(\psi_{=}\right)$foram determinadas pela relação entre o volume para cada teor de água e o volume inicial. Para o acompanhamento da redução do volume da massa, utilizouse um cilindro graduado de acrílico. O volume (V) de cada grão foi obtido por meio da medição dos três eixos ortogonais, comprimento (a), largura (b) e espessura (c), como proposto por MOHSENIN [15], em vinte grãos ao longo do processo de secagem, com auxilio de um paquímetro digital, de acordo com a seguinte expressão:

$\mathrm{V}=\frac{\pi \mathrm{abc}}{6}$

\section{4 - Análise estatística}

Os dados experimentais da porosidade, da massa especifica real e da massa específica aparente foram submetidos à análise de regressão, sendo selecionado o modelo matemático mais adequado para expressar a relação entre estas características e o teor de água da soja.

Aos dados experimentais de contração volumétrica unitária foram ajustados os modelos matemáticos linear, exponencial, BALA \& WOODS [2] adaptado, RAHMAN [16], CORREAA et al. [5] e equações de 4 a 8, respectivamente. Além destes modelos, para os dados de contração volumétrica da massa de grãos, foi ajustado o modelo de KROKIDA \& MAROULIS [10], equação 9. Para o ajuste dos modelos aos dados experimentais, foi utilizado o programa computacional Statistica $5.0 \AA$. A análise de regressão não linear foi realizada pelo método Quasi-Newton.

$\psi=\mathrm{A}+\mathrm{BU}$

$\psi=$ A.e BU* $^{\text {B }}$

$\psi=1-A\left(1-e^{-\beta\left(U_{0}^{*} \cdot U^{*}\right)}\right)$

$\psi=1+\beta \cdot\left(U^{*}-U_{0}^{*}\right)$

$$
\begin{aligned}
& \psi=\frac{1}{\mathrm{~A}+\mathrm{B} \cdot \mathrm{e}^{\mathrm{U}^{*}}} \\
& \psi=\frac{\mathrm{m}_{\mathrm{s}}}{\mathrm{V}_{\mathrm{mo}}} \cdot\left(\frac{1}{\rho_{\mathrm{aps}}}+\frac{\mathrm{B} \cdot \mathrm{U}^{*}}{\rho_{\mathrm{ag}}}\right)
\end{aligned}
$$

em que:

$\psi \quad$ - indice de contração volumétrica da massa granular, decimal

A, B - parâmetros que dependem do produto

U. - teor de água inicial do produto, decimal (b.s.)

$\beta \quad$ - coeficiente de contração volumétrica

$\mathrm{m}$ - massa seca do produto, $\mathrm{kg}$

$\mathrm{V}_{\mathrm{m}}$. - volume inicial da massa granular, $\mathrm{m}^{3}$

$\rho_{a, .} \quad$ - massa específica do produto seco, $\mathrm{kg} \mathrm{m}$

Os dados experimentais foram comparados com os valores calculados pelos modelos, por meio dos erros médios relativo $(\mathrm{P})$ e estimado (SE), conforme descrito a seguir:

$\mathrm{P}=\frac{100}{\mathrm{n}} \cdot \sum\left(\frac{|\mathrm{Y}-\hat{\mathrm{Y}}|}{\mathrm{Y}}\right)$

$\mathrm{SE}=\sqrt{\frac{\sum(\mathrm{Y}-\hat{\mathrm{Y}})^{2}}{\mathrm{GLR}}}$

em que:

n - número de observações experimentais

$\hat{Y} \quad$ - valor observado experimentalmente

$\hat{Y} \quad$ - valor calculado pelo modelo

GLR - graus de liberdade do modelo (número de dados menos o número de parâmetros do modelo)

Para análise do grau de ajuste de cada modelo foram consideradas as magnitudes do coeficiente de determinação ajustado (variância explicada), do erro médio relativo e estimado e a verificação do comportamento da distribuição dos resíduos.

\section{3 - RESULTADOS E DISCUSSÕES}

Na Figura 2, estão apresentados os valores experimentais e estimados da porcentagem de espaços vazios na massa de grãos de soja em função do teor de água.

Verifica-se, ainda, que a porosidade diminui linearmente de 44,7 para $41,1 \%$ com a redução do teor de água na faixa entre 0,31 a 0,15 (b.s.). Acima de 0,31 (b.s.) a porcentagem de espaços vazios entre grãos permanece praticamente constante. Esses valores de porosidade foram superiores àqueles observados por DESHPANDE, BAL \& OJHA [8], que verificaram redução da porosidade de 39,5 para 37,0\% quando o teor de água dos grãos de soja diminuiu de 0,25 para 0,08 (b.s.). Segundo MCMINN \& MAGEE [14], esta redução deve-se às modificações estruturais associadas às alterações celulares do produto, devido à retirada de água durante o processo de secagem. 


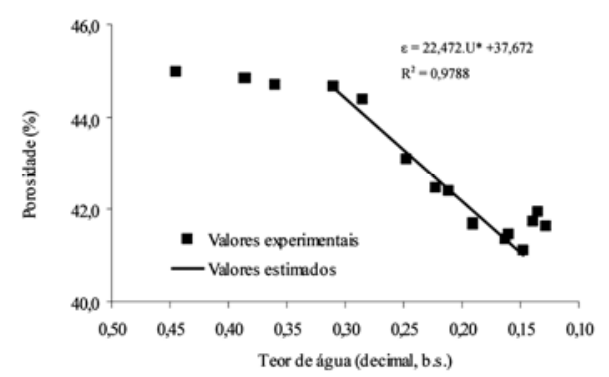

FIGURA 2 - Valores da porosidade calculados e estimados em função do teor de água dos grãos de soja

Nas Figuras 3 e 4 estão apresentados os valores experimentais e calculados pela composição química da massa específica real ( $\rho r_{n}$ e $\rho_{r 2}$ ), e da massa específica aparente $\left(\rho_{p_{1}}\right.$ e $\left.\rho_{p_{2}}\right)$ para diferentes teores de água, respectivamente.

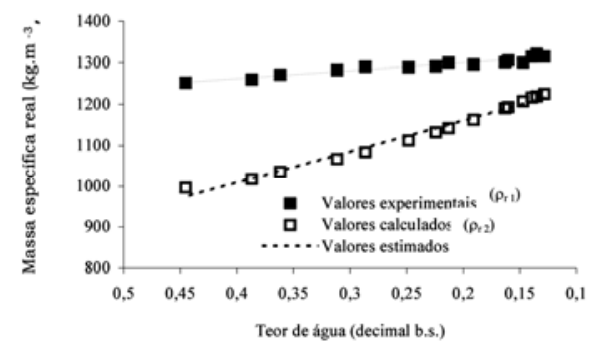

FIGURA 3 - Valores experimentais da massa específica real $\left(\rho_{\text {. }}\right)$ e calculados em função da composição química $\left(\rho_{\mathrm{r}}\right)$ dos grãos de soja para diferentes teores de água

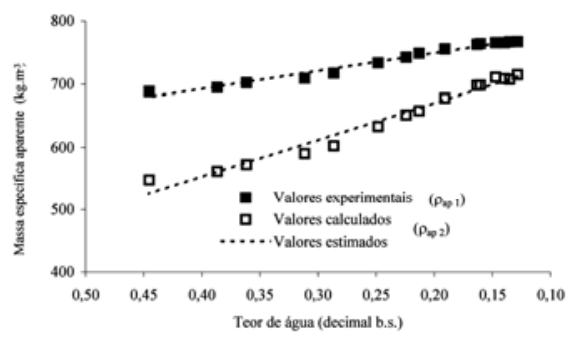

FIGURA 4 - Valores experimentais da massa específica aparente $\left(\rho_{x, y}\right)$ e calculados pela composição química $\left(\rho_{x, 2}\right)$ dos grãos de soja para diferentes teores de água

Verifica-se um aumento da massa específica real e da massa específica aparente com a redução do teor de água, como observado para a maioria dos produtos agrícolas, independentemente da metodologia utilizada. Observa-se que os valores das massas especificas, obtidos em função da composição química do produto, foram inferiores aos dados experimentais. No entanto, constata-se que as diferenças entre os valores experimentais e calculados diminuíram com a redução do teor de água. AFONSO JÚNIOR, CORREAA \& ANDRADE [1] calcularam a massa específica real em função da composição química para grãos de milheto e encontraram resultados superiores aos experimentais, contrário ao verificado para os grãos de soja no presente trabalho. Entretanto, CORREAA et al. [6] verificaram, para grãos de trigo, resultados semelhantes aos encontrados para soja.

$\mathrm{Na}$ Tabela 3 estão apresentados os modelos lineares de regressão ajustados aos valores experimentais e calculados de massa especifica real $\left(\rho_{\mathrm{r} 1}, \rho_{\mathrm{r} 2}\right)$ e massa especifica aparente $\left(\rho_{\mathrm{p}, 1}, \rho_{\mathrm{ap},}\right)$ e os coeficientes de determinação $\left(R^{2}\right)$ com seus respectivos niveis de significância. Verifica-se que o modelo linear representou satisfatoriamente os dados experimentais e calculados da massa específica real e aparente.

TABELA 3 - Modelos de regressão ajustados aos valores experimentais e calculados de massa especifica real e aparente e os coeficientes de determinação $\left(R^{2}\right)$ com seus respectivos níveis de significância

\begin{tabular}{lc}
\hline Equaçbes de regressao & $\mathrm{R}^{2}(\%)$ \\
Massa especifica real & $93,41^{*+}$ \\
\hline$\rho_{\mathrm{r} 1}=-189,37^{* *} \cdot\left(\mathrm{U}^{*}\right)+1337,6$ & $98,41^{*+}$ \\
\hline$\rho_{\mathrm{r} 2}=-762,32^{* *} \cdot\left(\mathrm{U}^{*}\right)+1313,9$ & \\
\hline Massa especifica aparente & $97,72^{*+}$ \\
\hline$\rho_{\mathrm{r} 1}=-283,91^{* *} \cdot\left(\mathrm{U}^{*}\right)+806,10$ & $97,45^{*+}$ \\
$\rho_{\mathrm{r} 2}=-585,67^{* *} \cdot\left(\mathrm{U}^{*}\right)+787,34$ & \\
\hline "Significativo a 1\% de probabilidade, pelo teste $\mathrm{F}$. & \\
**Significativo a 1\% de probabilidade, pelo teste t.
\end{tabular}

Na Tabela 4, estão apresentados os valores dos três eixos ortogonais (comprimento, largura, espessura) dos grãos de soja, utilizados para cálculo da contração volumétrica unitária para cada teor de água, bem como o volume calculado.

TABELA 4 - Dimensões médias dos eixos (a, b e c) ortogonais dos grãos de soja e o volume

\begin{tabular}{ccccc}
\hline \multirow{2}{*}{$\begin{array}{c}\text { Teor de água } \\
\text { (b.s.) }\end{array}$} & \multicolumn{3}{c}{ Eixos ortogonais (mm) } & Volume \\
\cline { 2 - 4 }$\left(\mathrm{mm}^{3}\right)$ & $\mathrm{a}$ & $\mathrm{b}$ & $\mathrm{c}$ & \\
\hline 0,445 & 8,57 & 6,78 & 5,53 & 168,26 \\
0,387 & 8,50 & 6,72 & 5,41 & 161,45 \\
0,362 & 8,51 & 6,74 & 5,41 & 162,41 \\
0,311 & 8,47 & 6,75 & 5,53 & 165,52 \\
0,287 & 8,48 & 6,73 & 5,45 & 162,92 \\
0,248 & 8,43 & 6,69 & 5,41 & 159,46 \\
0,224 & 8,38 & 6,70 & 5,46 & 160,43 \\
0,213 & 8,34 & 6,63 & 5,38 & 155,56 \\
0,191 & 8,28 & 6,67 & 5,44 & 157,43 \\
0,163 & 8,26 & 6,58 & 5,38 & 152,97 \\
0,160 & 8,20 & 6,59 & 5,35 & 151,34 \\
0,147 & 8,20 & 6,59 & 5,37 & 151,80 \\
0,139 & 8,15 & 6,56 & 5,36 & 150,05 \\
0,135 & 8,17 & 6,55 & 5,35 & 149,71 \\
0,128 & 8,14 & 6,53 & 5,37 & 149,26 \\
\hline
\end{tabular}

Na Tabela 5, encontram-se as equações de regressão para contração volumétrica da massa e unitária de grãos de soja, em função do teor de água, com seus respectivos coeficientes de determinação, erro médio relativo e estimado, e tendência de distribuição dos resíduos.

A análise dos dados indica que os modelos de BALA $\&$ WOODS [2] adaptado, RAHMAN [16], linear e exponen- 
cial ajustaram-se satisfatoriamente aos dados obtidos de contração volumétrica da massa, apresentando uma tendência menos acentuada de distribuição dos resíduos (distribuição aleatória). Observa-se, ainda, que estes modelos apresentaram coeficientes de determinação acima de $99 \%$ e baixos valores de erros médios relativos e estimados. Contudo, para apresentação dos resultados estimados de contração volumétrica da massa, optou-se pelo modelo linear, devido a sua simplicidade. Diversos autores já observaram que o modelo linear ajusta-se satisfatoriamente aos dados experimentais de contração volumétrica de vários produtos agrícolas $[1,11,12]$.

TABELA 5 - Modelos de contração volumétrica da massa de grãos de soja com seus respectivos coeficientes de determinação $(\mathrm{R})$, erros médios relativo $(\mathrm{P})$ e estimado $(\mathrm{SE})$, e tendência de distribuição dos resíduos

\begin{tabular}{|c|c|c|c|c|}
\hline $\begin{array}{c}\text { MODELos } \\
\text { Contração Volumétrica da Massa }\end{array}$ & $\mathrm{R}^{2}(\%)$ & P & SE & $\begin{array}{l}\text { Dišribuiç̧̃o dos } \\
\text { residuos }\end{array}$ \\
\hline$\psi_{\mathrm{m}}=1-1,3758 \cdot\left(1-\mathrm{e}^{-0,7571 \cdot\left(\mathrm{U}_{0}^{*} \cdot \mathrm{U}^{*}\right)}\right)$ & 99,85 & 0,3540 & 0,0037 & Aleatćria \\
\hline RAHMAN [16]: $\psi_{\mathrm{m}}=1 \cdot 0,9418 .\left(\mathrm{U}^{*} \cdot \mathrm{U}_{0^{*}}\right)$ & 99,64 & 0,5318 & 0,0055 & Aleatcóia \\
\hline$\psi_{\mathrm{m}}=476,0759 \cdot\left(0,0013+\frac{1,6561 \mathrm{U}^{*}}{1000}\right)$ & 97,69 & 1,4586 & 0,0140 & Tendenciosa \\
\hline CÓRREA et al. [5]: $\Psi_{\mathrm{m}}=\frac{1}{2,4894-0,9653 \mathrm{e}^{\mathrm{U}^{*}}}$ & 98,60 & 1,1365 & 0,0113 & Tendenciosa \\
\hline $\begin{array}{l}\text { Linear: } \psi_{m}=0,5866+0,9103 .\left(\mathrm{U}^{*}\right) \\
\text { Expon encial: } \psi_{\mathrm{m}}=0,6157 \mathrm{e} \\
1,0924 \cdot \mathrm{U}^{*}\end{array}$ & $\begin{array}{l}99,79 \\
99,82\end{array}$ & $\begin{array}{l}0,4245 \\
0,4254\end{array}$ & $\begin{array}{l}0,0044 \\
0,0041\end{array}$ & $\begin{array}{l}\text { Aleatćía } \\
\text { Aleatćria }\end{array}$ \\
\hline Contração Volumétrica Unitária & & & & \\
\hline $\begin{array}{l}\text { BALA \& WOODS [2] adaptado: } \\
\psi_{\mathrm{g}}=1+0,0340 \cdot\left(1-\mathrm{e}^{-4,6172 \cdot\left(\mathrm{U}_{0}^{*} \cdot \mathrm{U}^{*}\right)}\right)\end{array}$ & 89,75 & 0,8397 & 0,0123 & Aleatớia \\
\hline RAHMAN [16]: $\psi_{z}=1-0,3170,\left(\mathrm{U}^{*}-\mathrm{U}_{0}{ }^{*}\right)$ & 84,00 & 1,2583 & 0,0148 & Tendenciosa \\
\hline CÔRREA et al. [5]: $\psi_{\mathrm{m}}=\frac{1}{1,4192-0,2734 \mathrm{e}^{\mathrm{U}^{*}}}$ & 80,53 & 1,4450 & 0,0169 & Tendenciosa \\
\hline Linear: $\psi_{\mathrm{g}}=0,8555+0,3346 .\left(\mathrm{U}^{*}\right)$ & 84,28 & 1,2632 & 0,0152 & Tendenciosa \\
\hline Expon encial: $\psi_{\mathrm{g}}=0,8597 \mathrm{e}^{0,3505 . \mathrm{U}^{*}}$ & 83,56 & 1,3029 & 0,0155 & Tendenciosa \\
\hline
\end{tabular}

Para os dados de contração volumétrica unitária, o modelo de BALA \& WOODS [2] adaptado foi o que melhor se ajustou aos valores experimentais, apresentando uma tendência menos acentuada de distribuição dos resíduos (distribuição aleatória) e melhores coeficientes de determinação e erros médios relativos e estimados, conforme também observado por RIBEIRO et al. [18] para grãos de trigo.

Nas Figuras 5 e 6, apresenta-se a distribuição dos resíduos em função dos valores estimados para cada modelo matemático ajustado de contração volumétrica da massa e unitária, respectivamente.

Comprova-se, na Figura 5, que as distribuições de resíduos para os modelos de BALA \& WOODS [2] adaptado, RAHMAN [16], linear e exponencial apresentaram comportamento aleatório, indicando ser estes modelos apropriados para descrição da contração volumétrica da mas- sa de grãos de soja. No entanto, para os modelos de CORRÊA et al. [5] e KROKIDA \& MAROULIS [10], observa-se uma distribuição tendenciosa dos resíduos, sugerindo que estas equações representam de forma menos satisfatória o fenômeno estudado.
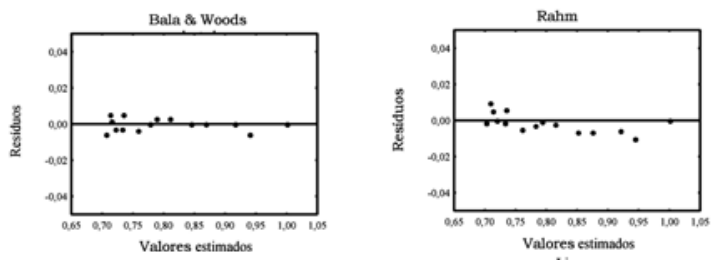

Exponencial
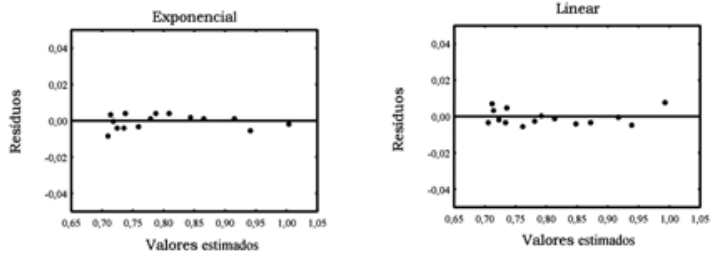

Corrêea
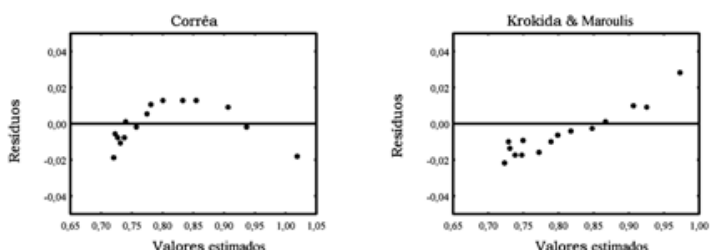

FIGURA 5 - Distribuição dos resíduos para cada modelo matemático ajustado em função dos valores estimados de contração volumétrica da massa de grãos de soja
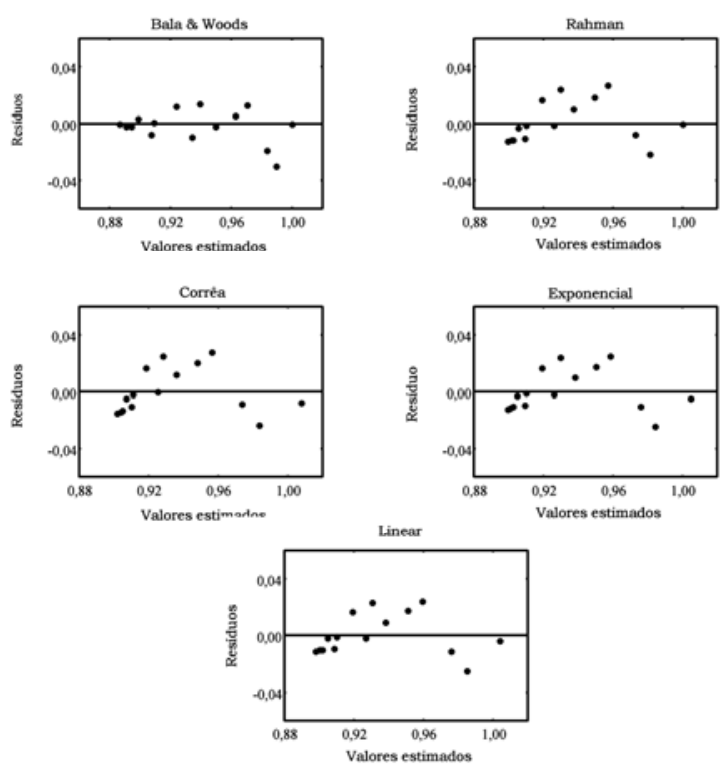

FIGURA 6 - Distribuição dos resíduos para cada modelo matemático ajustado em função dos valores estimados de contração volumétrica unitária de soja 
Na Figura 6, observa-se que a distribuição residual para o modelo de BALA \& WOODS [2] adaptado, apresenta comportamento aleatório, indicando ser este modelo apropriado para descrição da contração volumétrica unitária de soja, ao contrário dos demais, que apresentaram uma distribuição tendenciosa dos resíduos.

Nas Figuras 7 e 8 estão apresentados os valores experimentais de contração volumétrica unitária e da massa de grãos de soja ajustados pelo modelo de BALA \& WOODS [2] adaptado e linear, respectivamente, em função do teor de água.

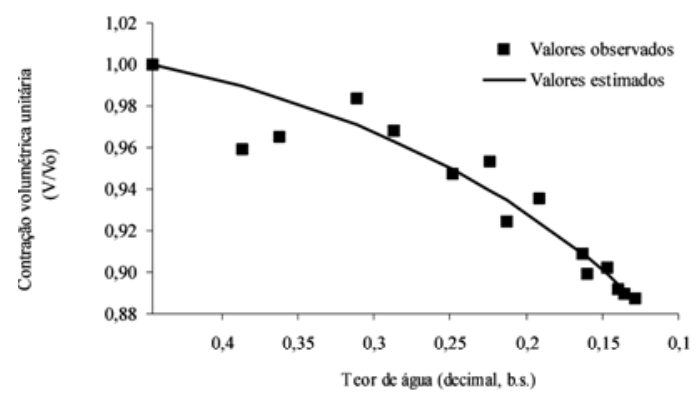

FIGURA 7 - Valores experimentais e estimados pelo modelo de BALA \& WOODS [2] adaptado de contração volumétrica unitária em função do teor de água

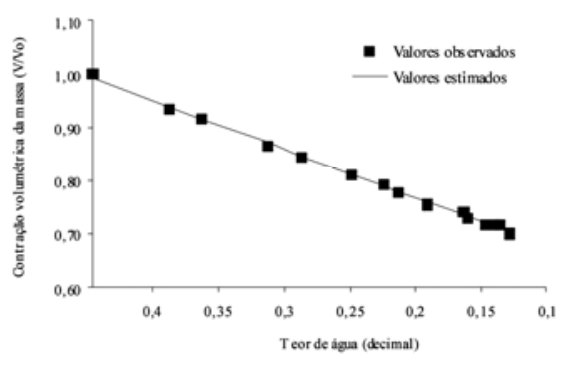

FIGURA 8 - Valores experimentais e estimados pelo modelo linear de contração volumétrica da massa de grãos de soja em função do teor de água

\section{4 - CONCLUSÕES}

Com base nos resultados obtidos, pode-se concluir que:

- a redução do teor de água provoca a diminuição linear da porosidade na faixa entre 0,31 e 0,15 (b.s.) e aumento das massas específicas aparente e real;

- os valores das massas específicas real e aparente, calculados a partir da composição química dos grãos de soja, são inferiores aos valores experimentais;

- os modelos de BALA \& WOODS [2] adaptado, RAHMAN [16], linear e exponencial são apropriados para descrição da contração volumétrica da massa de grãos de soja;

- o modelo BALA \& WOODS [2] adaptado é o mais apropriado para descrição da contração volumétrica unitária de soja.

\section{5 - REFERÊNCIAS BIBLIOGRÁFICAS}

[1] AFONSO JÚNIOR, P.C; CORREAA, P.C.; ANDRADE, E.T. Análise da variação das propriedades fisicas e contração volumétrica dos grãos de milheto (Pennisetum glaucum) durante o processo de dessorção. Revista Brasileira de Armazenamento. Viçosa, v. 25, n. 1, p. 15-2 1, 2000.

[2] BALA, B.K.; WOODS, J.L. Simulation of deep bed malt drying. Journal Agricultural Engineering Research, New York, v.30, n.3, p.235-244, 1984.

[3] BRASIL. Ministério da Agricultura e Reforma Agrária. Regra para análise de sementes. Brasília, DF: MARA, 1992.

[4] BROOKER, D. B., BAKKER-ARKEMA, F. W., HALL, C. W. Drying and storage of grains and oilseeds. Westport: AVI, 1992.

[5] CORREAA, P.C.; RIBEIRO, D.M.; RESENDE, O.; AFONSO JÚNIOR, P.C.; GONELI, A.L.D. Mathematical modelling for representation of coffee berry volumetric shrinkage. In: International Drying Symposium, 14th. Drying 2004, 2004a, São Paulo: Brazil, v.A, p.742-747.

[6] CORREA, P.C.; RIBEIRO, D.M.; RESENDE, O.; HENRIQUES, D.R.; SOUZA M.A. Análise da porosidade, massa específica aparente e real do trigo durante $o$ processo de secagem. Anais... XXXIII CONGRESSO BRASILEIRO DE ENGENHARIA AGRÍCOLA. São Pedro SP, 2004b. 1CD-ROM.

[7] COUTO, S.M.; MAGALHÃES, A.C.; QUEIROZ, D.M.; BASTOS, I.T. Massa específica aparente e real e porosidade de grãos de café em função do teor de umidade. Revista Brasileira de Engenharia Agricola e Ambiental, Campina Grande, v.3, n.1, p.61-68, 1999.

[8] DESHPANDE, S.D.; BAL, S.; OJHA, T.P. Physical properties of soybean. Journal of Agricultural Engineering Research, p. 89-98, 1993.

[9] HALL, C.W. Drying and storage of agricultural crops. Westport: AVI, 1980.

[10] KROKIDA, M.K.; MAROULIS, Z.B. Effect of drying method on shrinkage and porosity. Drying Technology, New York, v. 15, n. 10, p.2441-2458, 1997.

[11] LANG, W.; SOKHANSANJ, S. Bulk volume shrinkage during drying of wheat and canola. Journal of Food Process Engineering, Trumbull, v.16, n.4, p.305-314, 1993.

[12] LANG, W.; SOKHANSANJ, S.; ROHANI, S. Dynamic shrinkage and variable parameters in Bakker-Arkema's mathematical simulation of wheat and canola drying. Drying Technology, New York, v.12, n.7, p.1687-1708, 1994. 
[13] LEWIS, M.J. Propriedades físicas de los alimentos y de los sistemas de procesados. Zaragoza: Editorial Acribia S.A., 1993.

[14] MCMinN, W.A.M.; MAgeE, T.R.A. Physical characteristics of dehydrated potatoes part I. Journal of Food Engineering, London, v.33, n.1-2, p.37-48, 1997.

[15] MOHSENIN, N.N. Physical properties of plant and animal materials. New York: Gordon and Breach Publishers, 1986.

[16] RAHMAN, S. Physical properties of foods. Florida: CRC Press LLC, 1995. 500p.

[17] RATTI, C. Shrinkage during drying of foodstuffs. Journal of Food Engineering, London, v.23, n.1, p.91-105, 1994.

[18] RIBEIRO, D.M.; CORREA, P.C.; RESENDE, O.; GUIMARÃES, F.F.; COSTA, D.R. Forma, tamanho e contração volumétrica do trigo durante o processo de secagem. Anais... XXXIII CONGRESSO BRASILEIRO DE ENGENHARIA AGRÍCOLA. São Pedro SP, 2004. 1CDROM.
[19] RUFFATO, S.; CORRÊA, P.C.; MARTINS, J.H.; MANTOVANI, B.H.M.; SILVA, J.N. Influência do processo de secagem sobre a massa específica aparente, massa específica unitária e porosidade de milho-pipoca. Revista Brasileira de Engenharia Agricola e Ambiental, Campina Grande, v.3, n.1, p.45-48, 1999.

[20] SOKHANSANJ, S.; LANG, W. Prediction of kernel and bulk volume of wheat and canola during adsorpsion and desorption. Journal of Agricultural Engineering Research, New York, v.63, n.2, p. 129-136, 1996.

[21] TOWNER, G.D. The tensile stress generated in clay through drying. Journal Agricultural Engineering Research, New York, v.37, n.4, p.279-289, 1987.

[22] UNITED STATES DEPARTMENT OF AGRICULTURE USDA. Handbook of the nutritional contents of foods. New York: Dover Publications Inc., 1975.

[23] ZOGZAS, N.P.; MAROULIS, Z.B.; KOURIS, D.M. Densities, shrinkage and porosity of some vegetables during air drying. Drying Technology, New York, v.12, n.7, p.1653-1666, 1994. 